\title{
Reconhecimento de Caracteres Manuscritos Utilizando Sistemas Imunológicos Artificiais
}

\author{
Fernando P. A. Lima James C. Silva Amanda P. A. Lima Carlos R. Minussi \\ Departamento de Engenharia Elétrica, Faculdade de Engenharia de Ilha Solteira (FEIS) \\ Universidade Estadual Paulista "Júlio de Mesquita Filho" (UNESP), Ilha Solteira, SP, BRASIL \\ E-mails: engfernandoparra@gmail.com, jamesclauton@gmail.com, \\ amandaparra.eng@gmail.com, minussi@dee.feis.unesp.br
}

\begin{abstract}
Palavras-chaves: Reconhecimento de Caracteres Manuscritos, Processamento de Imagens, Sistemas Imunológicos Artificiais, Algoritmo de Seleção Negativa.
\end{abstract}

Resumo: Este artigo apresenta uma aplicação dos sistemas imunológicos artificiais para o reconhecimento de caracteres manuscritos. Tomando-se como base um processo biológico do sistema imunológico, utiliza-se uma adaptação do Algoritmo de Seleção Negativa para discriminar as amostras de imagens, obtendo uma classificação para os caracteres manuscritos. A principal aplicação deste método é auxiliar em processos de reconhecimento de padrões de imagens, visão computacional, bem como a tomada de decisão. Para avaliar este método, utilizou-se uma base de dados de imagens de caracteres manuscritos numéricos. Os resultados obtidos pelo método, quando comparado com a literatura especializada mostram precisão e eficiência.

\section{Introdução}

O reconhecimento de manuscritos (palavras ou caracteres) é um problema que cresceu nos últimos 20 anos, sendo objetivo de muitas pesquisas na área de reconhecimento de padrões. Isto porque, apesar do constante uso de tecnologias da informação, em muitas tarefas ainda utilizam documentos manuscritos, desta forma o reconhecimento de manuscritos se torna uma poderosa ferramenta para realizar análises de dados, autenticações, reconhecimento e classificação de padrões, e principalmente, tomada de decisões [12].

$\mathrm{O}$ reconhecimento de manuscritos se aplica diretamente em algumas classes de problemas de reconhecimento de padrões do cotidiano do ser humano, como por exemplo: autenticação de assinaturas em documentos, identificação de endereços em envelopes postais, indexação de documentos históricos, entre outros problemas. Para resolver estas classes de problemas é necessário desenvolver ferramentas tolerantes a variações de estilos de grafias, ruídos, etc., de maneira que a capacidade de reconhecimento seja condizente com a realidade da escrita humana.

Neste contexto, na literatura especializada existem várias propostas de sistemas inteligentes para reconhecimento de manuscritos, os quais apresentam bom desempenho e adaptabilidade aos problemas. A seguir apresentam-se os trabalhos mais relevantes.

Em [7] apresenta-se uma rede neural com técnica de treinamento multi escala para reconhecimento de manuscritos. Antes da análise das imagens utiliza-se a técnica de processamento de imagens de Thresholding para tratar as imagens. Um sistema computacional com interface gráfica desenvolvido em Delphi foi proposto em [8] para reconhecer imagens de dígitos utilizando uma rede neural multi-layer perceptron com treinamento via retropropagação.

Um algoritmo para reconhecimento e classificação de caracteres manuscritos foi apresentado em [3]. Este algoritmo se baseia em um princípio biológico do sistema imunológico, o processo de seleção clonal. Neste artigo os autores avaliaram o método com uma base de dados disponibilizada pelo repositório de dados UCI (University of California Irvine). No artigo [11] apresenta-se uma metodologia para o reconhecimento de caracteres manuscritos utilizando um sistema hibrido composto por duas redes neurais, sendo uma rede neural de base radial e uma rede neural multi-layer perceptron. Em [2] apresenta-se uma de 
neural de Kohonen com treinamento pelo algoritmo do neurônio vencedor para reconhecimento de caracteres manuscritos. Os caracteres utilizados neste trabalho estão no idioma Tamil.

Neste artigo, apresenta-se um método para reconhecimento de caracteres manuscritos utilizando sistemas imunológicos artificiais. A partir da leitura e processamento das imagens dos dígitos manuscritos, aplica-se o algoritmo de seleção negativa (ASN) [6] para reconhecer e classificar os caracteres. O algoritmo foi adaptado para classificar as imagens nas classes disponíveis utilizando a afinidade entre os padrões. Para avaliar o desempenho do método, foram realizados testes com uma base de dados real de caracteres manuscritos numéricos contendo 200 amostras.

Neste trabalho optou-se por utilizar sistemas imunológicos artificiais (SIA) devido as suas características naturais para o reconhecimento de padrões e por apresentarem bom desempenho e eficiência em outros tipos de problemas, conforme destacado em [9].

Este artigo está organizado como a seguir. Na seção 2 apresenta-se a base de dados utilizada nos testes. A descrição do algoritmo de seleção negativa (ASN) está na seção 3. Na seção 4 apresenta-se a metodologia proposta. As aplicações e resultados são apresentados na seção 5 e por fim, a seção 6 apresenta a conclusão para este trabalho.

\section{Base de Dados de Caracteres Manuscritos}

Para este trabalho foi construída uma base de dados de caracteres manuscritos numéricos contendo 200 amostras, sendo 20 amostras de cada tipo de padrão (dos números de 0 a 9). Os números foram manuscritos por uma pessoa em uma tabela, e esta tabela foi digitalizada, conforme ilustrado na Figura 1. Cada caractere manuscrito foi extraído com uma dimensão de $32 \times 32$ pixels e armazenado em formato BMP.

\begin{tabular}{|l|l|l|l|l|l|l|l|l|l|l|l|l|l|l|l|l|l|l|l|}
\hline 1 & 2 & 3 & 4 & 5 & 6 & 7 & 8 & 9 & 0 & 1 & 2 & 3 & 4 & 5 & 6 & 7 & 8 & 9 & 0 \\
\hline 1 & 2 & 3 & 4 & 5 & 6 & 7 & 8 & 9 & 0 & 1 & 2 & 3 & 4 & 5 & 6 & 7 & 8 & 9 & 0 \\
\hline 1 & 2 & 3 & 4 & 5 & 6 & 7 & 8 & 9 & 0 & 1 & 2 & 3 & 4 & 5 & 6 & 7 & 8 & 9 & 0 \\
\hline 1 & 2 & 3 & 4 & 5 & 6 & 7 & 8 & 9 & 0 & 1 & 2 & 3 & 4 & 5 & 6 & 7 & 8 & 9 & 0 \\
\hline 1 & 2 & 3 & 4 & 5 & 6 & 7 & 8 & 9 & 0 & 1 & 2 & 3 & 4 & 5 & 6 & 7 & 8 & 9 & 0 \\
\hline 1 & 2 & 3 & 4 & 5 & 6 & 7 & 8 & 9 & 0 & 1 & 2 & 3 & 4 & 5 & 6 & 7 & 8 & 9 & 0 \\
\hline 1 & 2 & 3 & 4 & 5 & 6 & 7 & 8 & 9 & 0 & 1 & 2 & 3 & 4 & 5 & 6 & 7 & 8 & 9 & 0 \\
\hline 1 & 2 & 3 & 4 & 5 & 6 & 7 & 8 & 9 & 0 & 1 & 2 & 3 & 4 & 5 & 6 & 7 & 8 & 9 & 0 \\
\hline 1 & 2 & 3 & 4 & 5 & 6 & 7 & 8 & 9 & 0 & 1 & 2 & 3 & 4 & 5 & 6 & 7 & 8 & 9 & 0 \\
\hline 1 & 2 & 3 & 4 & 5 & 6 & 7 & 8 & 9 & 0 & 1 & 2 & 3 & 4 & 5 & 6 & 7 & 8 & 9 & 0 \\
\hline
\end{tabular}

Figura 1: Base de dados

\section{Algoritmo de Seleção Negativa}

O algoritmo de seleção negativa (ASN) proposto por [6] para detecção de mudanças em sistemas é baseado na seleção negativa de linfócitos T dentro do timo. Este processo trabalha com a discriminação de células próprias e não-próprias. O algoritmo é executado em duas fases, conforme descrito a seguir [5]:

1. Censoriamento

a) Defina o conjunto de cadeias próprias (S) que se deseja proteger;

b) Gere cadeias aleatórias e avalie a afinidade (Match) entre cada uma delas e as cadeias próprias. Caso a afinidade seja superior a um limiar estipulado, rejeite a cadeia. Caso contrário, armazene-a em um conjunto de detectores (R).

2. Monitoramento 
a) Dado o conjunto de cadeias que se deseja proteger (cadeias protegidas), avalie a afinidade entre cada uma delas e o conjunto de detectores. Se a afinidade for superior a um limiar preestabelecido, então um elemento não-próprio é identificado.

Na figura 2 apresentam-se os fluxogramas das fases do ASN.

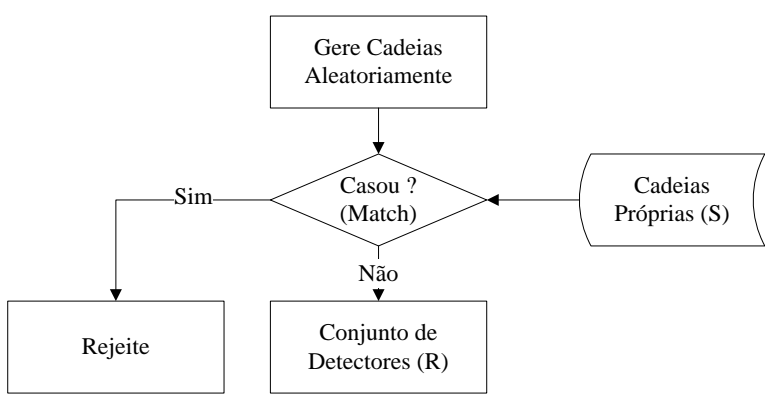

(a)

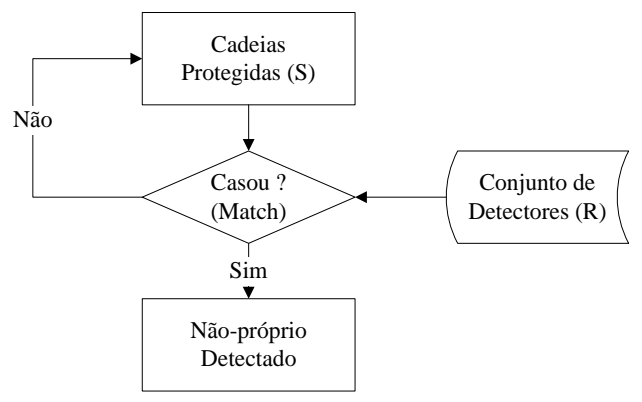

(b)

Figura 2: Fluxogramas do ASN.

A fase de censoriamento do ASN consiste-se basicamente em gerar um conjunto de detectores. Os detectores são análogos às células tipo $\mathrm{T}$ maturadas capazes de reconhecer agentes patogênicos. A fase de monitoramento consiste-se em monitorar um sistema, visando identificar uma mudança no comportamento do mesmo, e assim classificar esta mudança utilizando o conjunto de detectores criados na fase de censoriamento [4].

\subsection{Critério de Afinidade}

Para avaliar a afinidade entre as cadeias e comprovar que elas são semelhantes, utilizase um critério conhecido como casamento. O casamento pode ser perfeito ou parcial [9]. Neste trabalho utiliza-se o casamento parcial.

No casamento parcial, uma quantidade de variáveis entre os padrões deve ter o mesmo valor para se confirmar o casamento, sendo a quantidade (taxa de afinidade) definida previamente. A taxa de afinidade representa o grau de semelhança necessário para ocorrer o casamento entre os dois padrões. A taxa de afinidade é definida através da seguinte relação [1]:

$$
T A f=\left(\frac{A n}{A t}\right) * 100
$$

sendo:

TAf : taxa de afinidade;

An : número de cadeias normais no problema (cadeias próprias);

At : número total de cadeias no problema (cadeias próprias e não-próprias).

A equação (1) propõe uma relação estatística entre todas as amostras do problema para o cálculo da taxa de afinidade. A expressão (2) representa a forma de quantificar a afinidade total entre os padrões [1]:

$$
A f_{T}=\frac{\sum_{i=1}^{L} V c}{L} * 100
$$

sendo:

$A f_{T}: \%$ de afinidade entre os padrões analisados;

$L \quad$ : quantidade total de variáveis;

Vc : variáveis casada; 
$\sum_{i=1}^{L} V c:$ somatória (quantidade) de variáveis casadas.

Desta forma se $A f_{T}$ for maior ou igual a $T A f$ ocorre o casamento entre os dois padrões (são considerados semelhantes).

\section{Metodologia Proposta}

O sistema de reconhecimento de caracteres manuscritos apresentado nesta seção é baseado nos sistemas imunológicos artificiais [5], em especial no algoritmo de seleção negativa [6]. O método proposto se divide em três módulos: pré-processamento de imagens, censoriamento e o monitoramento dos dados.

$\mathrm{Na}$ sequência apresenta-se o pré-processamento das imagens e o algoritmo de reconhecimento de caracteres manuscritos.

\subsection{Pré-Processamento das Imagens}

Todas as imagens da base de dados apresentadas na seção 2 são pré-processadas utilizando técnicas de processamento de imagens, antes de serem analisadas pelo algoritmo de seleção negativa. As imagens inicialmente são binarizadas e posteriormente dilatadas conforme ilustrado na figura 3.

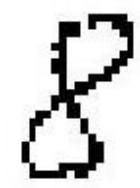

(a)

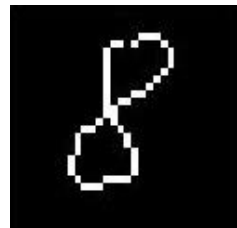

(b)

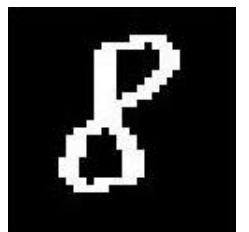

(c)

Figura 3: Pré-processamento das imagens (a) imagem original, (b) imagem binarizadas, (c) imagem dilatada.

\subsection{Algoritmo de reconhecimento de caracteres manuscritos}

O sistema de reconhecimento de caracteres manuscritos é composto por três módulos, sendo o pré-processamento de imagens (figura 3), o censoriamento (off-line) e o monitoramento (online). O diagrama de blocos do algoritmo é apresentado na figura 4.

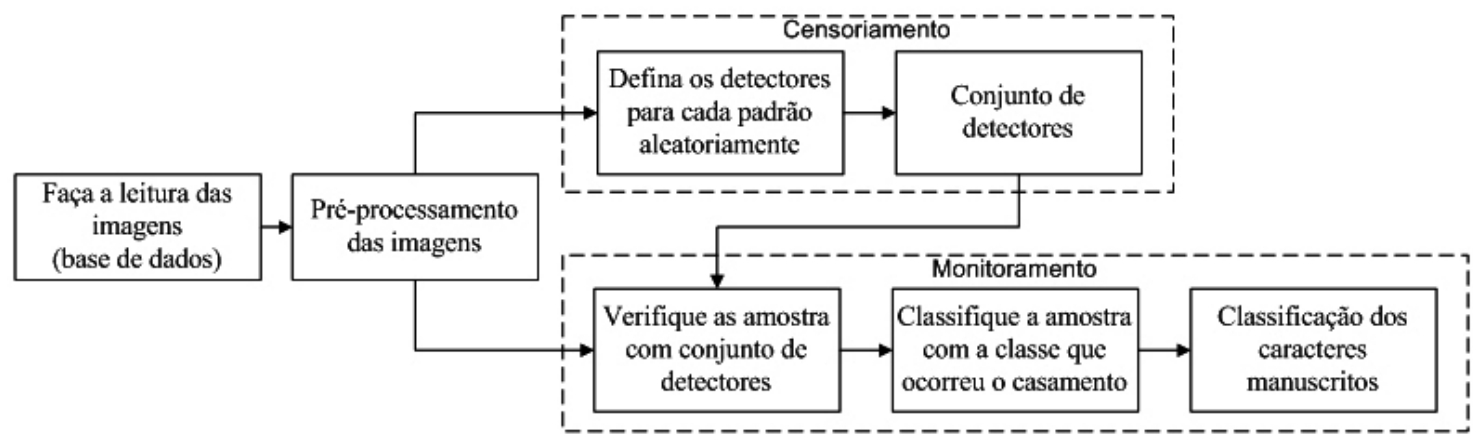

Figura 4: Diagrama de blocos do sistema.

O pré-processamento é realizado a fim de melhorar a qualidade das imagens e aumentar as características de cada padrão. 
No módulo de censoriamento são definidos aleatoriamente uma quantidade $n$ de detectores para cada padrão. Este conjunto de detectores é utilizado no processo de monitoramento para verificar o casamento em relação aos padrões em análise.

No monitoramento faz-se a verificação do casamento, avaliando a quantidade de pixels semelhante entre os padrões (quantificando a afinidade). Um casamento ocorre quando a afinidade entre os dois padrões é superior à taxa de afinidade definida. Quando ocorre um casamento entre os padrões, o padrão que se casou ao detector é automaticamente reconhecido e classificado com a classe pertencente ao padrão detector.

\section{Aplicações e Resultados}

Nesta seção apresentam-se os resultados obtidos com a aplicação da metodologia proposta na base de dados de caracteres manuscritos. Todos os testes foram realizados utilizando um PC Intel Core 2 Duo $1.9 \mathrm{GHz}, 2$ GB de Memória RAM, e sistema operacional Windows 7 Ultimate 32 bits. O algoritmo foi desenvolvido em MATLAB [10].

Nos testes realizados o objetivo foi avaliar a metodologia proposta, verificando a eficiência, precisão e o tempo computacional em relação a diferentes configurações do conjunto de detectores. Utilizou-se como parâmetro uma taxa com um valor fixo de $85 \%$. Foram gerados três conjuntos de detectores, sendo os conjuntos I, II e III que possuem 20, 40 e 60 padrões detectores, respectivamente. Os conjuntos de detectores gerados utilizam $10 \%, 20 \%$ e $30 \%$ das amostras, sendo que para cada configuração cada padrão utiliza 2, 4 e 6 detectores respectivamente.

$\mathrm{Na}$ Tabela 1 apresentam-se os resultados obtidos pelo sistema de reconhecimento de caracteres manuscritos, quando aplicado à base de dados.

Tabela 1 - Resultados obtidos pelo método.

\begin{tabular}{cccc}
\hline \multirow{2}{*}{ Classificação } & \multicolumn{3}{c}{ Conjunto de detectores } \\
\cline { 2 - 4 } & $I$ & $I I$ & $I I I$ \\
\hline $\begin{array}{c}\text { Amostras } \\
\text { testadas }\end{array}$ & 200 & 200 & 200 \\
\hline $\begin{array}{c}\text { Classificações } \\
\text { Corretas }\end{array}$ & 178 & 183 & 188 \\
\hline $\begin{array}{c}\text { Classificações } \\
\text { Erradas }\end{array}$ & 22 & 17 & 12 \\
\hline Acerto (\%) & $89 \%$ & $91,5 \%$ & $94 \%$ \\
\hline Tempo (ms) & 62,3 & 63,8 & 61,5 \\
\hline
\end{tabular}

Cada teste foi executado 20 vezes a fim de garantir a veracidade dos resultados. Foi possível observar que o sistema de reconhecimento de caracteres manuscritos apresenta um bom desempenho (índice de acerto igual a $94 \%$ na melhor configuração), e que a quantidade de detectores influência diretamente no reconhecimento e classificação final. Sugere-se utilizar $30 \%$ das informações da base de dados para gerar o conjunto de detectores, visando proporcionar robustez ao sistema. À medida que a quantidade de detectores aumenta, o sistema se torna mais preciso, isto porque quanto mais conhecimento, mais eficiente é o processo de reconhecimento e classificação.

$\mathrm{Na}$ Tabela 2 apresenta-se um estudo comparativo entre o método proposto e os principais métodos disponibilizados na literatura.

Tabela 2 - Análise comparativa de resultados.

\begin{tabular}{|l|c|c|c|c|}
\hline Referência & {$[8]$} & {$[11]$} & {$[7]$} & Este artigo. \\
\hline \% Acerto & $100 \%$ & $93,5 \%$ & $73,61 \%$ & $94 \%$ \\
\hline
\end{tabular}


Pode-se observar que o método proposto, neste trabalho, apresenta um bom desempenho em relação aos métodos disponíveis na literatura.

\section{Conclusão}

O algoritmo proposto apresentou excelentes resultados, obtendo um índice de acerto de 94\% para a melhor configuração do sistema em todas as amostras da base de dados. A fase de geração de detectores é a que demanda maior tempo computacional, no entanto é executada de forma off-line não acarretando prejuízo ao algoritmo. A fase de monitoramento do sistema, a partir da leitura dos dados, é realizada rapidamente, com tempo inferior a 65 milésimos de segundo, o que credencia o algoritmo a ser uma ferramenta utilizada em tempo real. Desta forma conclui-se que o sistema de reconhecimento proposto com base nos sistemas imunológicos é bastante eficiente, confiável, robusto quando aplicado nas amostras de dígitos manuscritos.

\section{Agradecimentos}

Os autores agradecem a FAPESP pelo apoio financeiro de pesquisa (Proc. № 2011/06394-5).

\section{Referências}

[1] D. W. Bradley and A. M. Tyrrell. Immunotronics - Novel Finite-State-Machine Architectures with Built-In Self-Test Using Self-Nonself Differentiation. IEEE Transactions on Evolutionary Computation.Vol. 6, pp. 227-238 (2002).

[2] P. Bunamathi and G. M. Nasira. Handwritten Tamil Characters Recognition Using Artificial Neural Networks, International Conference on Process Automation, Control and Computing (PACC), pp. 1-5 (2011).

[3] Y. C. Chunlin Liang; L. Peng and X. Zhong. A Handwritten Characters Recognition Algorithm based on Artificial Immune, International Conference on Computer Application and System Modeling (ICCASM 2010), pp. 273-276 (2010).

[4] D. Dasgupta. "Artficial Immune Systems and Their Applications". Springer-Verlag New York, Inc., Secaucus, NJ, USA, 1998.

[5] L. N. de Castro. "Engenharia Imunológica: Desenvolvimento e Aplicação de Ferramentas Computacionais Inspiradas em Sistemas Imunológicos Artificiais". Tese de Doutorado, Faculdade de Engenharia Elétrica e de Computação, Universidade Estadual de Campinas, Campinas, Brasil, 2001.

[6] S. Forrest; A. Perelson; L. Allen and R. Cherukuri. Self-Nonself Discrimination in a computer, Proc. do IEEE Symposium on Research in Security and Privacy, pp. 202-212 (1994).

[7] V. Ganapathy; and K. L. Liew. Handwritten Characters Recognition Using Multiscale Neural Network Training Technique, World Academy of Science, Engineering and Technology, Vol. 15, pp. 32-37 (2008)

[8] F. P. A. Lima; J. C. Silva and C. R. Minussi. Reconhecimento de Dígitos com uso de Redes Neurais Artificiais, Revista Omnia Exatas, vol. 3, nº 3, pp. 25-34 (2010).

[9] F. P. A. Lima. "Analysis of Voltage Disturbances in Distribution Electrical Systems Based on Artificial Immune Systems", Dissertation, University Estadual Paulista - UNESP, Ilha Solteira, March2013, 169 p.

[10] Matlab (2011). 7.8 Version, Mathworks Company.

[11] Y. Perwej and A. Chaturvedi. Machine Recognition of Hand Written Characters Using Neural Networks, International Journal of Computer Applications, vol. 14, nº 2, pp. 6-9 (2011).

[12] E. Vellasques. "Classificação de Pontos de Segmentação de Dígitos Manuscritos". Dissertação de Mestrado, Pontifícia Universidade Católica do Paraná, 2006. 